\title{
Artificial Neural Network Models for Rainfall Prediction in Pondicherry
}

\author{
Akash D Dubey \\ Department of Computing Science and Information System, \\ SMCS-CEST, \\ Fiji National University, Fiji
}

\begin{abstract}
Rainfall forecasting plays an important role in catchment management applications, the flood warning system being one of them. Rainfall forecasting is one of the most difficult tasks given the variability of space, time and other given conditions change rapidly. Over the years, with the evolution of the intelligent computing methods, many rainfall prediction methods have been proposed, Artificial Neural Network being one of the most prominent. Since the last decade, many researchers have proposed different artificial neural network models in order to create accurate rainfall prediction models. In this paper, different artificial neural networks have been created for the rainfall prediction of Pondicherry, a coastal region in India. These ANN models were created using three different training algorithms namely, feed-forward back propagation algorithm, layer recurrent algorithm and feedforward distributed time delay algorithm. The number of neurons for all the models was kept at 20 . The mean squared error was measured for each model and the best accuracy was obtained by feed-forward distributed time delay algorithm with MSE value as low as .0083.
\end{abstract}

\section{Keywords}

Rainfall Prediction, Pondicherry, Artificial Neural Network, Feedback Back propagation, Layer Recurrent, Distributed time delay network

\section{INTRODUCTION}

Quantitative rainfall forecast has been a challenging task for catchment management and flood management for a long time. The forecasting of the rainfall distribution spatially and temporally is important for water quality and quantity management. This quantitative forecasting can be readily used by the flood warning system to increase the lead time for warning. Besides, the qualitative forecasting of rainfall can be used for analyze many water quality problems beforehand. Basically, the rainfall forecasting methods have been categorized into two categories. The first approach involves modeling of the physical laws by the study of the rainfall processes. The basic problem encountered in this study is that this process may cease to be feasible since rainfall is an end product comprising of multiple complex atmospheric processes which differ both spatially and temporally. Secondly, for modeling of the approach, a large volume of calculations are needed which may limit this models' capacity.

The second approach for the rainfall prediction follows the path of pattern recognition in which an attempt is made to recognize the pattern with the help of the features affecting rainfall. In other words, this approach attempts to find the relevance between the spatial and temporal rainfall features using the historical data that have been collected over the years, probably from the meteorology department of the region. This approach is considered to be appropriate since we do not need thorough understanding of the physical laws and the data requirements in this approach is not as extensive as for a process model.

Artificial Neural Network has been one of the most frequently used computing methods for forecasting. The distinguishing features of artificial neural networks make it viable to be used in forecasting researches by the practitioners and the researchers as well. Some of the most important features of artificial neural networks which are ideal for forecasting are:

(a) Artificial neural networks are data-driven self-adaptive methods which learn from examples and discover the relationships among data which may be too complex to define. ANN work ideally for those problems in which the relationship may not be clear among the data but there is abundant data available.

(b) Artificial Neural Network mainly works on its generalization capability. This generalization capacity enables the network to handle the unseen data. In addition to it, this generalization also takes care of the noise present in the given data set. This feature of ANN is very beneficial for forecasting.

(c) ANNs are considered as universal functional approximators i.e. they can compute the value of any given function to any precision and this has been proven by Irie and Miyak [1] and Hornik et. al. [2].

In this paper, artificial neural networks have been used to create different models which predict the monthly rainfall of Pondicherry, a coastal region in India. The input data set comprises of parameters affecting rainfall such as minimum temperature, maximum temperature, vapor pressure, potential evapotranspiration and crop evapotranspiration. The results obtained from all the models were then compared to each other using the Mean Square Error (MSE) and sub sequentially the conclusions were drawn.

This paper has been divided into the following sections: Section 2 discusses the earlier works that have been done in prediction of rainfall and climate using pattern recognition system. Section 3 explains geographical study of Pondicherry. Section 3 summarizes the artificial neural network, it structure and mechanism. Section 4 explains the methodology and the input and output data sets used in artificial neural network. Section 5 discusses the results of the models and concludes the research work along with the future works.

\section{PREVIOUS WORKS}

The use of artificial neural network in weather forecasting was first initiated by $\mathrm{Hu}[3]$ and since then, artificial neural networks have been extended to various environment forecasting tasks. French et al in 1992 created a neural 
network to forecast two-dimensional rainfall in one hour advance [4]. Kalogirou et al estimated the missing rainfall data in Cyprus using ANN [5]. Wong et al used artificial neural networks along with fuzzy rule base to create predictive model for rainfall in Switzerland [6]. In 1999, Koizumi used the data from the meteorological department, radar and satellites to predict the rainfall which yielded better results than the conventional ones [7]. Tooth et al used ANN and K-nearest neighbor algorithm to predict the real time flood forecasting in Sieve River Basin, Italy [8]. A combination of scaled conjugate gradient algorithm and evolving fuzzy neural network ANN was used by Abraham et al to predict the rainfall time series in Kerela, India [9]. The authors used 87 years of monthly rainfall data for this purpose.

In 2001, Luk et al developed three different models for rainfall predictions and compared them [10]. These three models were developed using multilayer feed forward neural network, Elman partial recurrent neural network and time delay neural network. Lin et al in used a neural network with two hidden layers to predict the storm rainfall which produced decent results [11]. A rainfall prediction model for long monsoons in Kerela was developed by Krishnakutty which produced results better than the statistical methods used [12]. Somvanshi et al developed a rainfall prediction model which made use of the past observations of Hyderabad (India) region [13]. Chattopadhyay developed a three layer neural model which used the data ranging from 1950 to 1995 to predict the average monsoon rainfall in India [14].

Hung et al developed a neural network that could predict the rainfall in real time in Bangkok [15]. This model was experimented in real world scenario where the data was fed using the hourly data of four years from 75 different weather stations. Panagiotis et al used artificial neural networks to predict the rain intensity in Athens, Greece [16]. A combination of Artificial Neural Networks and wavelet decomposition was used by Charaniya et al to predict the monthly rainfall with the use of the historical data [17]. ElShafie et al developed and compared two static and one dynamic neural network for the monthly and weekly rainfall prediction in Malaysia [18]. Deshpande used a multilayer perceptron neural network to predict the rainfall using the government authorized data in Yavatmal, Maharashtra, India [19].

\section{GEOGRAPHICAL STUDY OF THE REGION}

The area taken for our study was Pondicherry. Pondicherry is situated on the Coromandel coast between $11^{\circ} 45^{\prime}$ and $12^{\circ} 03^{\prime}$ $\mathrm{N}$ latitudes and $79^{\circ} 37^{\prime}$ and $79^{\circ} 53^{\prime} \mathrm{E}$ longitudes with an area of 293 sq. km. Pondicherry comprises of four districts Pondicherry, Mahe, Yanam and Karaika which have a total of 164 inhabited villages.

The normal annual rainfall in Pondicherry is $1272.7 \mathrm{~mm}$ as detected by the meteorological department of India. Pondicherry receives $62 \%$ of its rainfall in the form of cyclonic storms in the Northeast monsoon due to the cyclonic depression in the Bay of Bengal while the southwest monsoons contribute $26 \%$ of the rainfall, with November being the month of highest rainfall.

\section{ARTIFICIAL NEURAL NETWORKS $(\mathrm{ANN})$}

An artificial neural network (ANN) models the structure and the functional aspects of biological neural networks mathematically. There are an interconnected group of artificial neurons which processes the information using a connectionist approach. The ability of the neural networks to learn is modeled in the same way in which the human brain works. The neural networks are a complex adaptive system which changes its own internal structure with the help of the information which is flowing through it. Generally, these changes can be done by altering the weights which is assigned to each neuron.

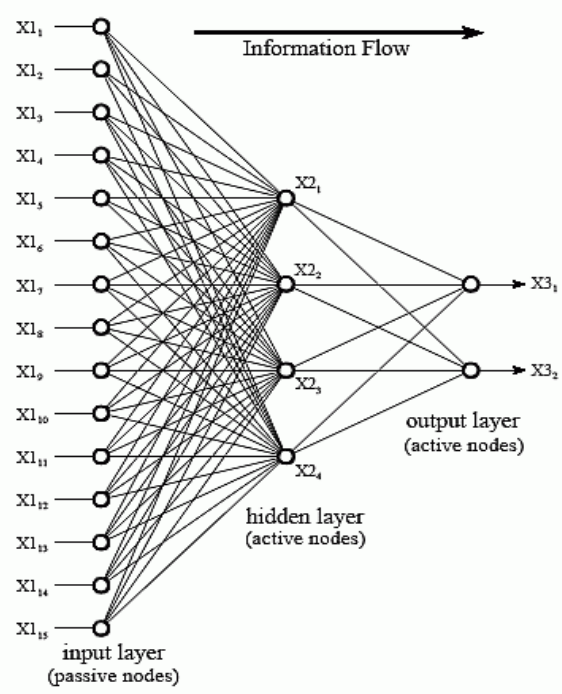

Figure 1. General Architecture of Artificial Neural Network

The neurons in the artificial neural networks are connected by synapses. This combination of neurons and synapses produce a value which is dependent on the values presented to them at their inputs. There are various types of neuron which generate their output based on different processes:

- Input neurons: The input values are fed to a network at the input neurons. The output of an input neuron is equal to the input value assigned to it.

- Hidden (sigmoid) neurons: The hidden neurons are responsible for the calculations that are performed within the network. The output of a sigmoid neuron is equal to the sum of its inputs multiplied by a sigmoid activation function.

- Output neurons: The output values of a given are available in the output neurons..

- Bias neurons: Bias neurons are mainly used to provide a constant bias to the other neurons and output a steady value of 1 .

Along with the neurons, there two functions which determine the behavior of a unit in the neural network, which normally remains constant for all the neurons in the network. The functions are Input function and Output/Activation function.

\section{RESEARCH METHODOLOGY}

Input and Output Data: For this study, five different parameters which are related with rainfall of a region were taken. These five parameters were minimum temperature, maximum temperature, water vapor pressure, potential evapotranspiration and crop evapotranspiration. A dataset of 1200 data was collected which extended from 1901 to 2000. For the training purposes, 800 samples were taken while 
validation and testing phase had 200 samples each. The input data sample for training was thus $5 \times 800$ matrix while the output layer data was 1 x800 matrix.

Madden and Williams have proved that there are significant large scale correlations between the minimum \& maximum temperature and the rainfall for North America and Europe [20]. This relation was globally expanded by Trenberth and Shea in 2005 [21]. The ratio of precipitation and potential evapotranspiration is known as Climate ratio which decides the climate type of a given place. The region under our study has humid climate and therefore this climate ratio is considered to be more than 1.2. In other words, potential evapotranspiration is directly related to the rainfall a place receives. Jinping Zhang et al have used the Fourier analysis to prove that there exists a complex relationship between the crop evapotranspiration and rainfall which may be related to El Nino, air-sea intersection and solar activity [22]

Normalization: The data collected for input layer were different in units and had no correlation among them. Therefore, to solve this problem, min-max normalization was used to limit the data values between 0 and 1 . According to the min-max normalization, each value was normalized using following equation:

$$
\operatorname{Normalized}\left(e_{i}\right)=\frac{e_{i}-E_{\min }}{E_{\max }-E_{\min }}
$$

There were 12 different ANN models which were trained and tested using different combinations of the training algorithms, training functions and adaptive learning functions. The training algorithms used in these models were feed forward back propagation algorithm, layer recurrent algorithm and feed forward distributed time-delay network. The training functions used were trainlm and trainrp while the adaptive learning functions were traingd and traingdm.

\section{RESULTS, DISCUSSION AND FUTURE WORKS}

The artificial neural network models were trained using the input data. There were total 12 ANN models which were trained using the 800 data samples of the region which were collected over 100 years. After the training, the validation and testing of the neural networks were done using 200 data samples each. The performance measure of the neural networks was the Mean Square Error(MSE). For a network to be more accurate, the MSE has to be as low as possible.

While training and testing the neural networks using the feed forward back propagation algorithm, the minimum MSE obtained was 0.0201 which occurred when the training function was TRAINLM and the adaptive learning function was LEARNGDM. When the neural networks were trained using the layer recurrent algorithms, the minimum MSE obtained was 0.0129 which was obtained when the training function was TRAINRP and the adaptive learning function is LEARNGDM. The feed forward distributed time delay network gave a minimum MSE of 0.0083 when the training function was TRAINLM and adaptive learning function was kept as LEARNGDM.

The details and the performance of each neural model under study has been summarized in the table 1 . From the table, it can be deduced that the best performance was delivered by the neural networks which were trained by the feed forward distributed time delay network in which the MSE ranged from 0.0083 to 0.0120 . Layer recurrent networks gave better results than the feed forward back propagation networks but were not better than the feed forward distributed time delay networks.

The figure 2, 3 and 4 shows the performance of the three best performing models i.e. model 9,10 and 11 during the training. The figure 5, 6 and 7 are the charts which show the testing results. These figures show the comparative charts of the actual rainfall that occurred during the given span of time and the predicted rainfall by the best performing neural networks. Model 11, whose MSE was 0.0083 has the best accuracy which is evident in the figure 7 where the two lines(actual rainfall and predicted rainfall) are almost on the same trend and values most of the time.

The network models which have been used in this research paper can be effectively used for the prediction of the missing values of rainfall at any given place. The feed-forward distributed time delay network can prove beneficial for this purpose since this network uses the sequential data for the training. Moreover, this research work can also be extended to predict the short term rainfall at a region which can help the rain crop harvesting the flood management system.

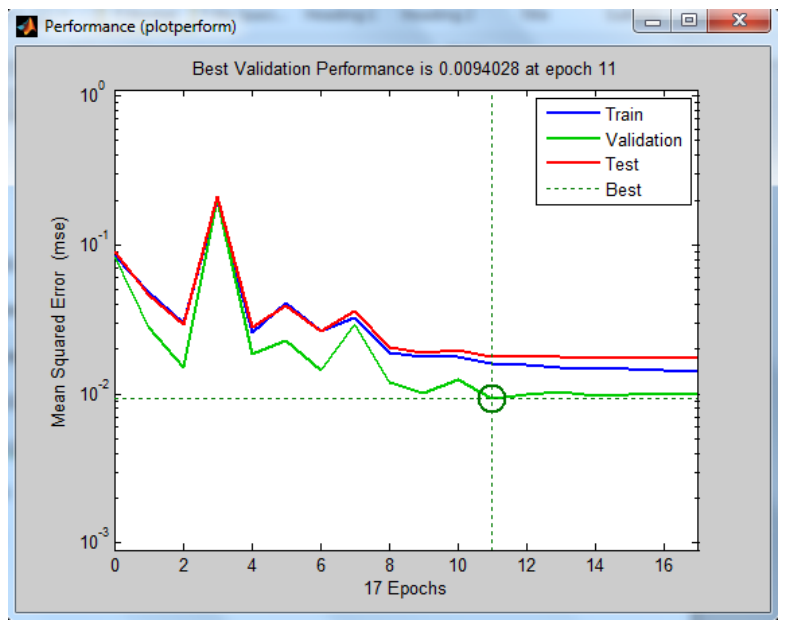

Figure 2 Model 9 Performance

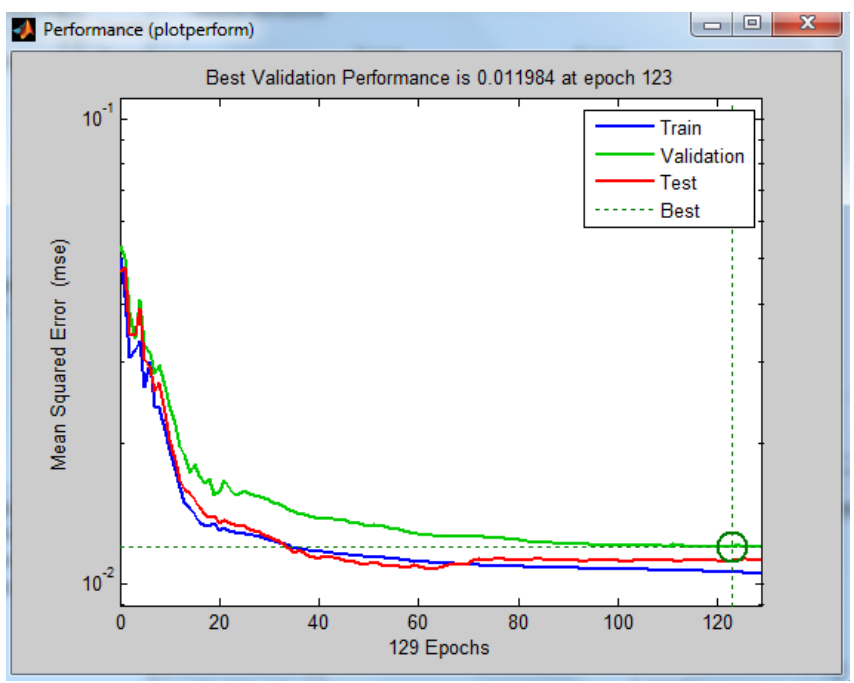

Figure 3 Model 10 Performance 


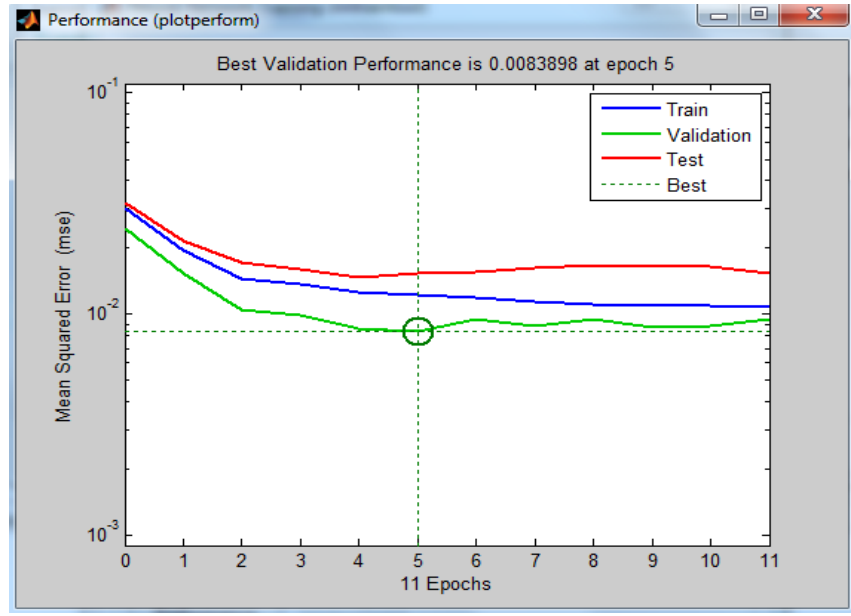

Figure 4 Model 11 Performance

Table 1: Artificial Neural Network Models and their performance

\begin{tabular}{|c|c|c|c|c|}
\hline Model\# & Training Algorithm & Training Function & $\begin{array}{l}\text { Adaptive Learning } \\
\text { Function }\end{array}$ & $\begin{array}{c}\text { Mean Square Error } \\
\text { (MSE) }\end{array}$ \\
\hline Model 1 & $\begin{array}{l}\text { Feed forward Back } \\
\text { Propagation }\end{array}$ & TRAINRP & LEARNGD & 0.0743 \\
\hline Model 2 & $\begin{array}{l}\text { Feed forward Back } \\
\text { Propagation }\end{array}$ & TRAINRP & LEARNGDM & 0.0232 \\
\hline Model 3 & $\begin{array}{l}\text { Feed forward Back } \\
\text { Propagation }\end{array}$ & TRAINLM & LEARNGD & 0.0419 \\
\hline Model 4 & $\begin{array}{l}\text { Feed forward Back } \\
\text { Propagation }\end{array}$ & TRAINLM & LEARNGDM & 0.0201 \\
\hline Model 5 & Layer Recurrent & TRAINRP & LEARNGD & 0.0140 \\
\hline Model 6 & Layer Recurrent & TRAINRP & LEARNGDM & 0.0129 \\
\hline Model 7 & Layer Recurrent & TRAINLM & LEARNGD & 0.0476 \\
\hline Model 8 & Layer Recurrent & TRAINLM & LEARNGDM & 0.0133 \\
\hline Model 9 & $\begin{array}{l}\text { Feed forward distributed } \\
\text { time delay }\end{array}$ & TRAINRP & LEARNGD & 0.0094 \\
\hline Model 10 & $\begin{array}{l}\text { Feed forward distributed } \\
\text { time delay }\end{array}$ & TRAINRP & LEARNGDM & 0.0119 \\
\hline Model 11 & $\begin{array}{l}\text { Feed forward distributed } \\
\text { time delay }\end{array}$ & TRAINLM & LEARNGD & 0.0083 \\
\hline Model 12 & $\begin{array}{l}\text { Feed forward distributed } \\
\text { time delay }\end{array}$ & TRAINLM & LEARNGDM & 0.0120 \\
\hline
\end{tabular}

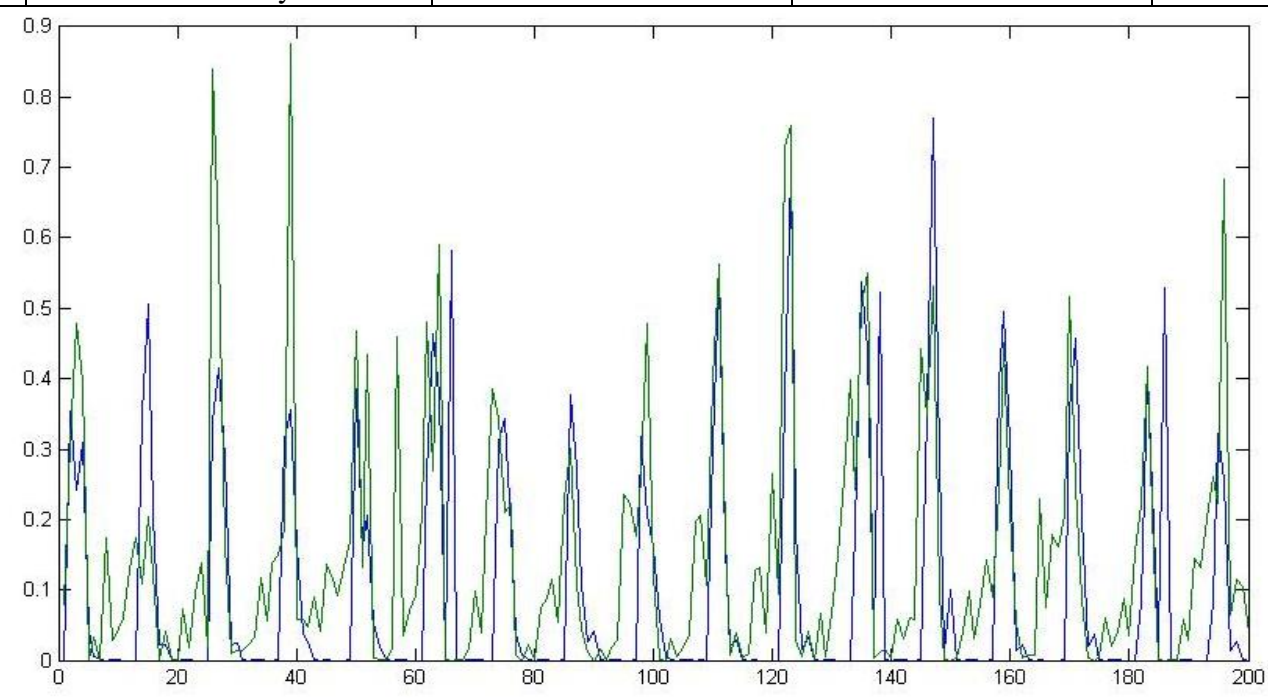

Figure 5: Model 9 Comparison Between Actual and Predicted data 


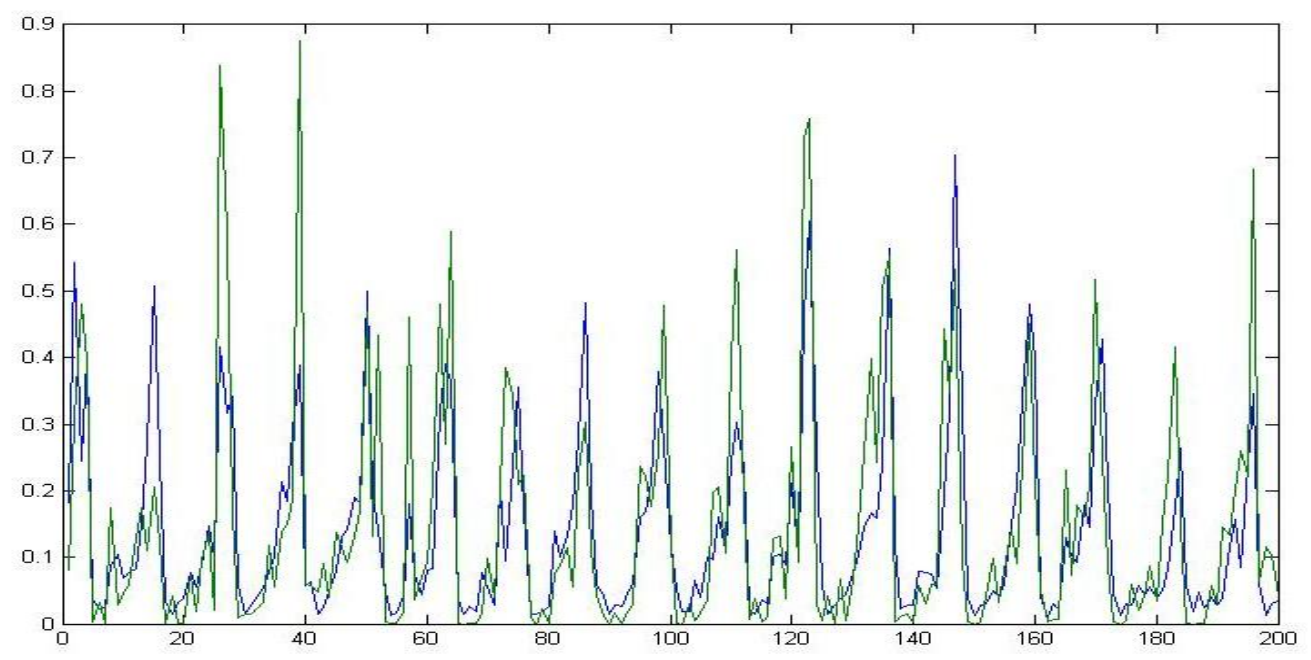

Figure 6: Model 10 Comparison Between Actual and Predicted data

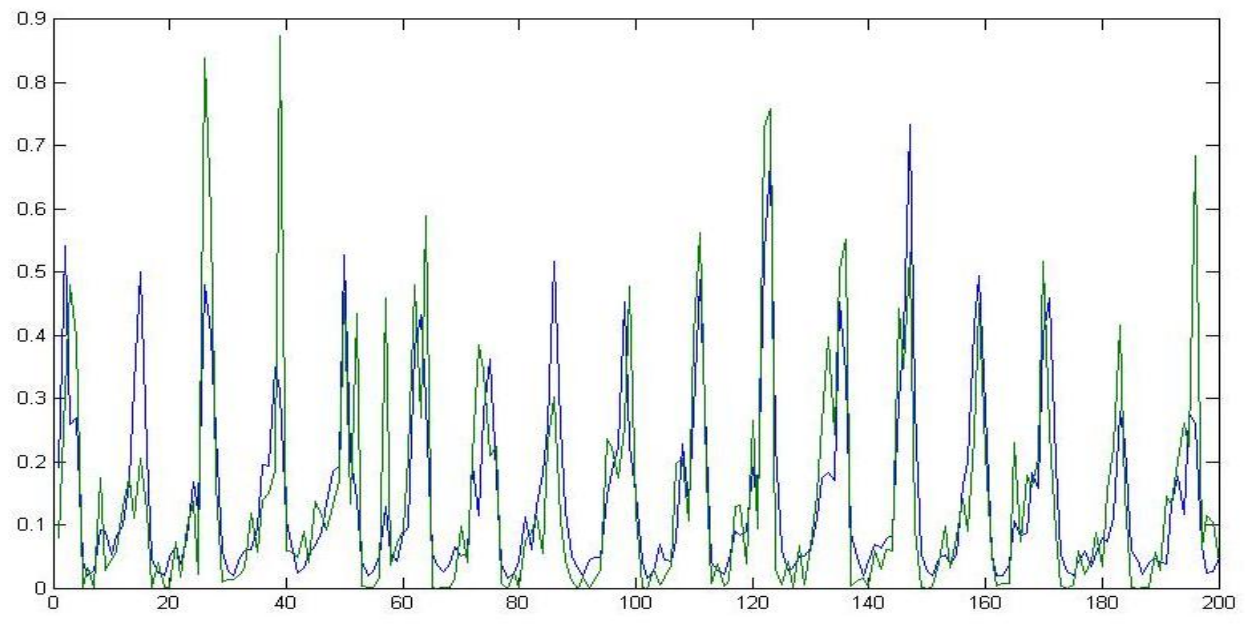

Figure 7: Model 11 Comparison Betweeen Actual and Predicted data

\section{REFERENCES}

[1] Irie, Bunpei, and Sei Miyake. Capabilities of threelayered perceptrons. Neural Networks, 1988., IEEE International Conference on. IEEE, 1988.

[2] Hornik, Kurt, Maxwell Stinchcombe, and Halbert White. Multilayer feedforward networks are universal approximators. Neural networks 2.5 (1989): 359-366.

[3] Hu, Michael Jen-Chao. Application of the adaline system to weather forecasting. Diss. Department of Electrical Engineering, Stanford University. 1964.

[4] French, Mark N., Witold F. Krajewski, and Robert R. Cuykendall. Rainfall forecasting in space and time using a neural network. Journal of hydrology 137.1 (1992): 131.

[5] Kalogirou, S. A., Neocleous, C., Constantinos, C. N., Michaelides, S. C. \& Schizas, C. N. A time series construction of precipitation records using artificial neural networks. Proceedings of EUFIT ' 97 Conference, 8-11 September, Aachen, Germany. pp 2409-2413 1997.
[6] Wong, K. W., Wong, P. M., Gedeon, T. D. \& Fung, C. C. Rainfall Prediction Using Neural Fuzzy Technique. 1999.

[7] Koizumi, K.: An objective method to modify numerical model forecasts with newly given weather data using an artificial neural network. Weather Forecast 14, 109-118, 1999.

[8] E.Toth, A.Brath, A.Montanari. Comparison of short-term rainfall prediction models for real-time flood forecasting. Journal of Hydrology 239 (2000) 132-147.

[9] Abraham, Ajith, Dan Steinberg, and Ninan Sajeeth Philip. Rainfall forecasting using soft computing models and multivariate adaptive regression splines. IEEE SMC Transactions, Special Issue on Fusion of Soft Computing and Hard Computing in Industrial Applications (2001).

[10] Luk, Kin C., James E. Ball, and Ashish Sharma. An application of artificial neural networks for rainfall forecasting. Mathematical and Computer modelling 33.6 (2001): 683-693. 
[11] Lin, Gwo-Fong, and Lu-Hsien Chen. Application of an artificial neural network to typhoon rainfall forecasting. Hydrological Processes 19.9 (2005): 1825-1837.

[12] Krishnakutty, N. Long-range monsoon rainfall prediction of 2005 for the districts and sub-division Kerala with artificial neural network. Current Science 90.6 (2006): 773.

[13] Somvanshi, V. K., Pandey, O. P., Agrawal, P. K., Kalanker, N. V., Prakash, M. R., \& Chand, R.. Modeling and prediction of rainfall using artificial neural network and ARIMA techniques. J. Ind. Geophys. Union 10.2 (2006): 141-151.

[14] Chattopadhyay, Surajit. Feed forward Artificial Neural Network model to predict the average summer-monsoon rainfall in India. Acta Geophysica 55.3 (2007): 369-382.

[15] Hung, N. Q., Babel, M. S., Weesakul, S., \& Tripathi, N. K. An artificial neural network model for rainfall forecasting in Bangkok, Thailand. Hydrology and Earth System Sciences 13.8 (2009): 1413-1425.

[16] Nastos, P., Moustris, K., Larissi, I., \& Paliatsos, A. Rain intensity forecast using Artificial Neural Networks in Athens, Greece. EGU General Assembly Conference Abstracts. Vol. 12. 2010.
[17] Charaniya, Nizar Ali, and Sanjay V. Dudul. Committee of artificial neural networks for monthly rainfall prediction using wavelet transform. Business, Engineering and Industrial Applications (ICBEIA), 2011 International Conference on. IEEE, 2011.

[18] El-Shafie, A., et al. Dynamic versus static neural network model for rainfall forecasting at Klang River Basin, Malaysia. Hydrology and Earth System Sciences Discussions 8.4 (2011): 6489-6532.

[19] Deshpande, Rohit R. On The Rainfall Time Series Prediction Using Multilayer Perceptron Artificial Neural Network. (2012): 1.

[20] Madden, Roland A., and Jill Williams. The correlation between temperature and precipitation in the United States and Europe. Monthly Weather Review 106.1 (1978): 142-147.

[21] Trenberth, Kevin E., and Dennis J. Shea. Relationships between precipitation and surface temperature Geophysical Research Letters 32.14 (2005).

[22] Zhang, J., Ding, Z., Yuan, W., \& Zuo, Q. Research on the relationship between rainfall and reference crop evapotranspiration with multi-time scales. Paddy and Water Environment 11.1-4 (2013): 473-482. 